\title{
Special issue: Highlights from the flagship Latin American Symposium on Circuits and Systems: selected papers from LASCAS 2013
}

\author{
Carlos Silva-Cardenas • Eduard Alarcón • \\ Sergio Bampi
}

Published online: 21 December 2014

(c) Springer Science+Business Media New York 2014

It is a pleasure and a privilege to present to the readers of the Springer Journal on Analog Integrated Circuits and Signal Processing (ALOG) a selection of the papers from the 4th edition of the Latin America Symposium on Circuits and Systems-LASCAS 2013, held in February 2013 in the historical Peruvian city of Cuzco.

The LASCAS Symposium is the premier event in circuits and systems in that region of the Americas. The papers selected by the Guest Editors fall in the focus of this Journal, and are among the $10 \%$ top quality of the 155 papers submitted to LASCAS 2013, and were selected by rigorous reviewing. While LASCAS contemplates applications and digital systems, most of the papers in this Special Issue address analog circuits and systems, the themes of interest to ALOG Journal readers.

From the 119 papers which were accepted and published in the IEEE LASCAS Proceedings, a total of 27 works were nominated and invited to submit a substantially improved manuscript on the same subject to this Special

C. Silva-Cardenas

Maestria de Ingenieria de las Telecomunicaciones, Pontificia

Universidad Catolica del Peru, Ave. Universitaria 1801,

Lima 32, Peru

e-mail: csilva@pucp.edu

E. Alarcón

Department of Electronics Engineering, Technical University of Catalunya (UPC BarcelonaTech), Office 105, Building C4, Campus Nord UPC, Calle Gran Capità s/n, 08034 Barcelona, Spain

e-mail: eduard.alarcon@upc.edu

\section{S. Bampi $(\bowtie)$}

Informatics Institute, Federal University of Rio Grande do Sul, Ave. Bento Goncalves 9500 - Setor 4, Porto Alegre 91501-970, Brazil

e-mail: bampi@inf.ufrgs.br
Issue of LASCAS 2013. The quality screening took advantage of the reviews and paper ratings for each of those 119 papers, which were narrowed down to 23 extended manuscripts. These went through a second-round of peer-review process and 14 papers, substantially extended versions from the LASCAS Symposium, were finally selected for this Special Issue, as the highlights from the flagship Latin American Symposium on Circuits and Systems. The papers in this Special Issue come from the Americas (North and South) and Europe.

The 14 selected papers cover a wide range of topics, from analog circuits design at ultra-low voltages, to amplifiers design, integrated circuits, wireless, and digital systems design, and up to topics at the application of communications and arithmetic circuits.

The first 3 papers in the Special Issue deal with low voltage analog CMOS design. The paper "Fully Integrated Inductive Ring Oscillators Operating at VDD below $2 k T /$ $q$ " by M. Machado et alli presents oscillators with two inductive loading schemes to work under extremely low DC supplies. H. Hernandez et alli propose and show in their paper a silicon implementation of "A fully Integrated Boost Converter For Thermoelectric Energy Harvesting" which upconverts from very low voltage supplies. The paper "Sub-1 V Band-Gap Based and MOS ThresholdVoltage Based Voltage References in $0.13 \mu \mathrm{m}$ CMOS" by D.M. Colombo et alli explores and compares low-voltage DC reference circuits with different circuit approaches possible in standard CMOS, compared to diode-based BGRs. Two papers on DC and instrumentation amplifiers designs target improvements on either DC gain or CMRR. H. Gomez-Ortiz introduces a "Robust to PVT Enhanced DC Gain Amplifier Using No Miller Capacitor Feedforward Compensation" in his paper, where a feedforward scheme improves DC gain and common mode range. 
Authors R. Abdullah and E. Sanchez-Sinencio in their paper on "A Biopotential Amplifier with Dynamic Capacitor Matching for Improved CMRR" demonstrate advances in techniques for dynamic matching to input in instrumentation amplifiers. The paper "A Configurable Analog Buffer Dedicated to a Wafer-Scale Prototyping Platform" by Y. Laflamme-Mayer et alli demonstrates in silicon the design of an analog buffer for digital systems that presents a degree of performance configuration. M.J. Barragan et alli present a low-overhead, programmable and low power on-chip harmonic signal generator with low distortion in their paper "On-Chip Sinusoidal Signal Generation with Harmonic Cancelation for Analog and Mixed-Signal BIST Applications". Circuits for wireless communications are focused in 3 papers in this Special Issue: in the work on "Very Small Inductorless 2nd Derivative Gaussian IRUWB Transmitter Module Using n/p-Latches as PDs" the authors L.C. Moreira et alli show the circuits for pulse shaping necessary in ultra-wideband transmitters. An integrated Doherty RF power amplifier design for constant power-added efficiency is demonstrated by M. L. Carneiro et alli in their paper "Fully Integrated Doherty Power Amplifier in CMOS $65 \mathrm{~nm}$ with Constant PAE in Backoff". Wireless communication systems design is also featured in this issue: co-authors J. Gaxiola-Sosa and K. Entesari present the "Design and implementation of a Wireless Medical System Prototype for Implantable Applications" accomplishing full-system demonstration for the communication with implanted medical devices in the ISM band. The authors I. S. A. Bezzam et alli present a key analog design contribution for digital circuits synchronization: the distribution of a system-clock in a resonant fashion. Their paper "Wide Operating Frequency Resonant Clock and Data Circuits for Switching Power Reductions" shows the buffering scheme used for reducing the system power budget in the clock distribution network.

The second group of papers in this issue is concerned with digital circuits and systems design, and applications, which are themes invited in the LASCAS calls for papers. Co-authors J. E. Guerrero-Ramirez et alli propose a semisystolic architecture to execute the QR method to compute eigenvalues on symmetric matrices in the paper "Hardware Design of an Eigensolver Based on the QR Method". Their design both in software and in HDL mapped to FPGA shows one order of magnitude speed up of the latter. G. Sanchez and co-authors explore in "Hardware-Friendly HEVC Motion Estimation: New Algorithms and Efficient VLSI Designs Targeting HD Videos" the implementation of motion estimation algorithms with respect to their regularity and performance potential in hardware implementation, using the HEVC software and the EPZS algorithm as references. The last paper by $W$. Jose et alli deals with a full design space exploration at the architecture level of multi-cores dedicated to matrix algebra in the work "Algorithm-Oriented Design of Efficient Many-Core Architectures applied to Dense Matrix Multiplication".

We would like to express our appreciation to the Circuits and Systems Society of the IEEE for sponsoring LASCAS, to Dr. Mohammed Ismail, Editor-in-chief of the Journal, for the opportunity to organize this Special Issue, and to the assistance of Raja Chinnadurai, from the Springer Journal Editorial Office, for the diligent work in the managing functions at their site.

We are grateful to the competent volunteer work of all LASCAS 2013 reviewers and of Special Issue reviewers, who assured a sound technical review of more than 155 papers previously submitted. We hope you appreciate the quality of this Special Issue.

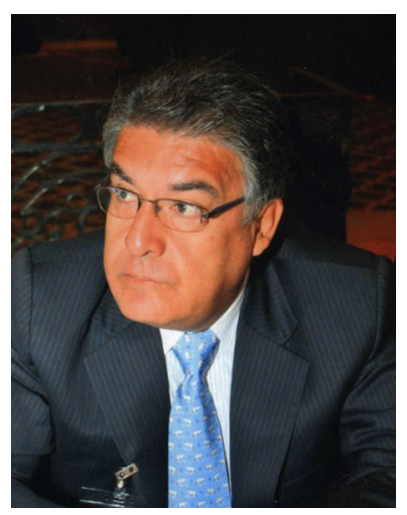

Carlos Silva-Cardenas received the B.Sc. from Universidad Nacional de Ingeniería, Perú; M.Sc. and $\mathrm{Ph} . \mathrm{D}$. degrees in Informatic engineering from Autonomous University of Barcelone, Spain. $\mathrm{He}$ is a IEEE Senior Member, was the founding chair and current chair of CAS IEEE Peru Section (2007), Chair of IEEE Peru Section (2009-2010), Vice-Chair of IEEE Peru Section (2007-2008; 1995-1997). He is Professor and Director of Master on Telecommunications at the Pontificia Universidad Católica del Perú and Director-founder of Microelectronic Research Group (1992). He is author of the first peruvian digital integrated circuit and he was Researcher at the Centro Nacional de Microelectrónica, Barcelone (1997-2001). He has taught courses for masters and doctoral level at: Universidad Autonoma Barcelona (2003), Universidad de Valencia-Spain (2004), Universidad Complutense de Madrid (2005) and Universidad Nacional de Tucumán, Argentine (2006 and 2008). He was visitant-researcher in INPG, TIMA Labs on Grenoble, France (Nov. 2007-Feb. 2008) and Analog and Mixed Signal Center on Texas A\&M University, USA (January-February 2014). Author and coauthor of 3 books about digital circuits design, 2 book chapters and 62 papers. He has been General Chair in 8th Latin American Test Workshop (Cusco, 11-14 March 2007) and XIII Workshop IBERCHIP (Lima, 14-16 March 2007), Programm chair of IV IEEE ANDESCON (2008) and I IEEE Latin American CAS (LASCAS, Iguazu, 2010), Geneal Chair of Second IEEE CAS Summer school (Cusco, January 2011) and General Chair of 2013 IV IEEE LASCAS (Cusco, 27th February-1st March) and he has coordinated 32 international and nationals congress and workshops. He has been General Secretary of the Iberoamerican Project on microelectronic: IBERCHIP (1997-2006). Silva-Cardenas is member of IEEE Prize Paper/Scholarship Awards Committee and CASS-BoG member during 2014 and he has received important prizes: National Prize of Engineering (CONIMERA, 1993), Prize: Encouraging creativity in science and technology (CONCYTEC, 1994) and Technological creativity Prize (Peruvian Telephone Company, 1993). He received the Award: Recognition for research by the Pontificia Universidad Catolica del Peru last 5 years: 2010-2014. Finally, the work carried out by SilvaCardenas in front of the IEEE CAS in Peru had led to three awards from IEEE: IEEE CASS 2010 Chapter of the Year Award and twice IEEE CASS 2011, IEEE CASS 2012 Region 9 Chapter of the Year Award. 


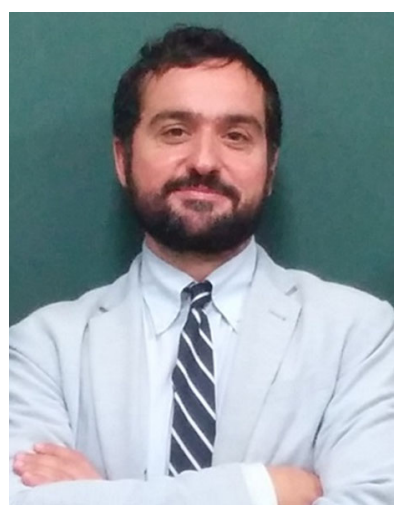

Eduard Alarcón received the M.Sc. (National award) and $\mathrm{Ph} . \mathrm{D}$. degrees (honors) in Electrical Engineering from the Technical University of Catalunya (UPC BarcelonaTech), Spain, in 1995 and 2000, respectively. Since 1995 he has been with the Department of Electronic Engineering at UPC, where he became Associate Professor in 2000. From August 2003 to January 2004, July to August 2006 and July to August 2010 he was a Visiting Professor at the CoPEC center, University of Colorado at Boulder, US, and during January-June 2011 he was Visiting Professor at the School of ICT/ Integrated Devices and Circuits, Royal Institute of Technology (KTH), Stockholm, Sweden. During the period 2006-2009 he was Associate Dean of International Affairs at the School of Telecommunications Engineering, UPC. He has co-authored more than 250 scientific publications, 5 books, 6 book chapters and 8 patents, and has been involved in different National, European and US (DARPA, NSF) R\&D projects within his research interests including the areas of on-chip energy management circuits, energy harvesting and wireless energy transfer, and nanotechnology-enabled wireless communications. He has given 25 invited or plenary lectures and tutorials in Europe, America and Asia, was appointed by the IEEE CAS society as distinguished lecturer for 2009-2010 and lectures yearly MEAD courses at EPFL. He has participated in Evaluation Boards for research proposals both in Europe (Chist-ERA, Belgium, Ireland, Italy) America (Canada) and Asia (Korea). He is elected Vice President of Finantial Activities of the IEEE CAS Society (2015-2016), twice member of the IEEE CAS Board of Governors (2010-2013, 2014) and member of the IEEE CAS long term strategy committee. He was recipient of the Myril B. Reed Best Paper Award at the 1998 IEEE Midwest Symposium on Circuits and Systems. He was the invited co-editor of a special issue of the Analog Integrated Circuits and Signal Processing journal devoted to current-mode circuit techniques, a special issue of the International Journal on Circuit Theory and Applications, and invited associate editor for a IEEE TPELS special issue on PwrSOC. He co-organized special sessions related to on-chip power management at IEEE ISCAS03, IEEE ISCAS06 and NOLTA 2012, and lectured tutorials at IEEE ISCAS09, ESSCIRC 2011, IEEE VLSI-DAT 2012 and APCCAS 2012. He was the 2007 Chair of the IEEE Circuits and Systems Society Technical Committee on Power Circuits. He was the technical program co-chair of the 2007 European Conference on Circuit Theory and Design-ECCTD07 and of LASCAS 2013, Special Sessions co-chair at IEEE ISCAS 2013, tutorial co-chair at ICM 2010, Demo Chair of BodyNets
2012, track co-chair of IEEE ISCAS 2007, IEEE MWSCAS07, IEEE ISCAS 2008, ECCTD'09, IEEE MWSCAS09, IEEE ICECS'2009, ESSCIRC 2010, PwrSOC 2010, IEEE MWSCAS12 and TPC member for IEEE WISES 2009, WISES 2010, IEEE COMPEL 2010, IEEE ICECS 2010, IEEE PRIME 2011, ASQED 2011, ICECS 2011, INFOCOM 2011, MoNaCom 2012, LASCAS 2012, PwrSOC 2012, ASQED 2012, IEEE PRIME 2012, IEEE iThings 2012 and CDIO 2013. He served as an Associate Editor of the IEEE Transactions on Circuits and Systems-II: Express briefs (2006-2007) and Associate Editor of the Transactions on Circuits and Systems-I: Regular papers (2006-2012) and currently serves as Associate Editor Elsevier's Nano Communication Networks journal (2009-), Journal of Low Power Electronics (JOLPE) (2011-) and in the Senior Editorial Board of the IEEE Journal on Emerging and Selected Topics in Circuits and Systems (2010-).

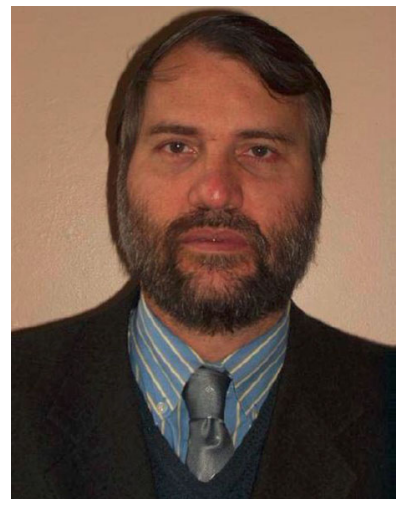

Sergio Bampi received the B.Sc in Electronics and the B.Sc. in Physics from the Federal Univ. of Rio Grande do Sul (UFRGS, 1979), and the M.Sc. (1982) and Ph.D. (1986) degrees in electrical engineering from Stanford University. He is a full professor at the Informatics Institute at UFRGS University, coordinator of the Microelectronics Design group. He served from 2005 to 2008 as the Technical DirectorCTO of the Microelectronics Center CEITEC in Brazil. Sergio Bampi served a consulting role for the Brazilian Microelectronics Program and industrial policies related to the electronics industry. Since 1988 he is a Ph.D. advisor and project leader in the Microelectronics and Computer Science Graduate Programs at UFRGS. His research interests are in the areas of CMOS IC design and modeling, dedicated complex architectures and ASICs for image and video processing, nano-MOSFET devices, mixed signal and RF CMOS design, and low power digital methodologies. He has co-authored more than 300 papers in these fields and in MOS devices, circuits, technology and CAD. Sergio Bampi was the President of the Research Funding agency FAPERGS in Brazil (2001-2002), President of the Brazilian Microelectronics Society (2002-2004), and Coordinator of the Graduate Program on Microelectronics at Federal University UFRGS (2003-2007). He chaired IEEE SBCCI (1997, 2005), LASCAS (2013), SBMICRO $(1989,2000)$ and Ibero-American Microelectronics (1995) conferences, and was track Chair at IEEE ICM, IFIP/IEEE VLSI-SoC, and IEEE ICECS Conferences. He is a member of IEEE, SBMICRO, IEEE Circuits and Systems, IEEE Solid-State Circuits, and Computer Societies. 